\title{
Review of: "Chemical, physical, and functional properties of Thai indigenous brown rice flours"
}

\author{
Rhowell Jr. Tiozon ${ }^{1}$ \\ 1 De La Salle University
}

Potential competing interests: The author(s) declared that no potential competing interests exist.

This study revealed Thai indigenous brown rice flours' chemical, physical, and functional properties, namely Khai Mod Rin and Noui Khuea. Findings include amylose, extractable phenolic, DPPH activity, bulk density, water adsorption capacity, solubility, and swelling properties. The research provided some information about these indigenous brown rice samples, but a further improvement on the result's discussion is needed. The following are the concerns and recommendations in this study.

1. The description of the "commercial rice flour (CMRF)" used was not further specified. I was able to imply that it is milled rice from the properties but, it should be directly stated. Since the indigenous rice samples are brown rice, the comparison should be with the brown rice also.

2. Besides using the iodine test, the samples can also be subjected to size exclusion chromatography to determine the amylose content, the chain length distribution, and the degree of polymerization of starch. With this, the researchers can better understand the specific use of these indigenous rice flours.

3. The researchers reasoned that they did not extract the bound component because it is not a pigmented rice. However, many researchers have extracted the free and bound components of brown rice. Refer to the following link:

1. https://doi.org/10.1111/tpj.15182

2. 10.1016/j.foodchem.2017.09.077

3. https://doi.org/10.1515/ijfe-2015-0346

In extracting the bound component of rice, the researchers can relate the dietary fiber composition of brown rice and bound phenolic concentration in their local benefit in the colon.

4. The FT-IR analysis can be complemented with NMR in future studies to compare the compounds elucidated.

5. Future investigations may relate the amylose-lipid complexes that can be obtained from the Differential Scanning Calorimeter in relation to their oil adsorption capacity.

6. Some of the discussions are out of place-for instance, the discussion on the rice storage proteins. The researchers also wrote that the indigenous rice flours could be the key source of minerals but did not produce any elemental analysis. Generally, the discussion has been chiefly pointing out related literature 
and not expanding on the results. Hence, further experiments should have been performed, and mechanisms should be further derived to contribute novel ideas.

7. Researchers can further improve conclusions by giving recommendations instead of arriving at statements that were not extensively proven in the experiment. 